\title{
The era of anti-vascular endothelial growth factor (VEGF) drugs in ophthalmology, VEGF and anti-VEGF therapy
}

\author{
DOROTA POŻAROWSKA ${ }^{1}$, PIOTR POŻAROWSKI \\ ${ }^{1}$ Chair of Ophthalmology, Department of Diagnostics and Microsurgery of Glaucoma, Medical University of Lublin, Poland \\ ${ }^{2}$ Department of Clinical Immunology, Medical University of Lublin, Poland
}

\begin{abstract}
Angiogenesis is a clue process for tissue development and function, both in normal and pathological conditions. This process is regulated by multiple molecular systems. One of the most potent is vascular endothelial growth factor (VEGF) and its receptor (VEGFR) system. Members of this family are involved in new vessel formation in embryogenesis and maturation, as well as in reparative or pathological reactions in later stages. They play a substantial role in regeneration, inflammation, wound healing, as well as in cancer pathology. Nowadays it is possible to modulate VEGF-VEGFR interactions in many pathological conditions using anti-VEGF therapy. This therapy has already achieved a grounded position in the management of rheumatological disorders, tumour progression, and metastasis. Such drugs as bevacizumab, ranibizumab, aflibercept, and pegaptanib have also proven to be very effective in the treatment of several ocular diseases, such as age-related macular degeneration (AMD), macular oedema, or proliferative retinopathies and iris neovascularisation. The indications for the application of this therapy in ophthalmology are becoming wider and wider. It may also be used for corneal pathologies and in anti-glaucoma procedures.
\end{abstract}

Key words: safety, VEGF, macular oedema, anti-VEGF therapy, AMD.

(Cent Eur J Immunol 2016; 41 (3): 311-316)

\section{Introduction}

Angiogenesis is a clue process for tissue development and function, both in normal and pathological conditions. Abundant factors and systems such as: vascular endothelial growth factors and receptors (VEGF-VEGFRs), angiopoietin-Tie receptor axis, Ephrin-Eph receptor pathway, insulin-like growth factor-1 (IGF-1), interleukin 8 (IL-8), platelet derived growth factor (PDGF), the Delta-Notch pathway, and Netrins-UNC5R pathway are involved in the angiogenesis process $[1,2]$. One of the most potent is the VEGF-VEGFR system [3]. These signalling molecules regulate vasculogenesis during developmental embryogenesis and angiogenesis in later stages, as in wound healing, tumour growth, inflammation, and ocular diseases $[2,4]$. In the eye VEGF is an important factor of ocular homeostasis [5]. It may be produced not only by vascular endothelial cells or pericytes, but also by retinal neurons and astrocytes, Müller cells, retinal pigment epithelium, and even by non-pigmented ciliary epithelium [5, 6]. Hypoxia is a proven stimulus for VEGF upregulation [5, 7]. This occurs by inducing transcriptional activator hypoxiainducible factor- $1 \alpha$ (HIF- $1 \alpha)$ and its target genes: nitric oxide synthase (NOS) or VEGF genes [7, 8]. As is well documented, hypoxia is an generative factor in many ocular diseases such as ocular ischaemic syndrome, proliferative retinopathies, and neovascular glaucoma. Hypoxia promotes VEGF transcription, so it upregulates angiogenesis to restore oxygen and nutrition supply for tissues [9]. However, VEGF not only plays the role in the angiogenesis and vascular barrier breakdown; it was shown that VEGF enhances leukocyte adhesion to vascular walls and increases expression of vascular cell adhesion molecule-1 (ICAM-1) in the retina and the brain [7]. Thus it plays a role in inflammation activation [7]. It was proven that VEGF is produced and acts only locally, so VEGF plasma concentrations do not correlate either with the in situ synthesis or action [5].

VEGF family in humans consist of several proteins: VEGF-A, VEGF-B, VEGF-C, VEGF-D, and placental growth factor (PlGF) $[1,3,10]$. All of them act by binding with specific receptors that are typical tyrosine kinase receptors (TKRs) [2, 4]. VEGF-A is a main factor for physiological and pathological angiogenesis [4]. It is a heparin-binding homodimeric glycoprotein of $36-46 \mathrm{kDa}$

Correspondence: Piotr Pożarowski, MD, PhD, Department of Clinical Immunology, Medical University of Lublin, Chodźki 4 A,

20-093 Lublin, Poland, e-mail: ppozarowski@yahoo.com

Submitted: 31.12.2015; Accepted: 22.01.2016 
weight $[11,12]$. It acts via VEGFR-1 (Flt1) and VEGFR-2 (KDR) receptors. The effects of their interactions are: stimulation of endothelial cells, migration of macrophages, increased vascular permeability, and a retinal blood barrier breakdown [4, 11]. Stimulation of VEGFR-1 (Flt1) enhances inflammatory cell (macrophages) attraction and migration [4]. VEGFR-2 is expressed mostly on vascular endothelial cells [13]. Its stimulation is a major signal not only for endothelial cell proliferation, migration, and angiogenesis, but also for proliferation of fibroblasts $[1,2]$. Moreover, it is thought that VEGF-A plays an important role in neural tissue because neurons express both VEGFR-1 and VEGFR-2. In vitro studies revealed that VEGF-A may have neurodevelopmental, neurotrophic, and neuroprotective roles [14]. Soluble forms: sVEGFR-1 (sFlt1) and sVEGFR-2, act as endogenous VEGF-A inhibitors $[3,15]$.

The gene of human VEGF is composed of eight exons and seven introns between them $[5,11]$. Due to alternative splicing of encoding VEGF-gene there are several isoforms of VEGF A: VEGF-A121, VEGF-A145, VEGF-A165, VEGF-A189, and VEGF-A206, which are classified by the number of amino acids $[2-4,11,16,17]$. Among them VEGF-A165 is the most potent and the most abundant one $[4,11,18]$. It acts by binding to both VEGFR-1 and VEGFR-2, and it induces angiogenesis, cell migration, and vascular permeability [4]. VEGF-A121 promotes endothelial cell proliferation, but also as VEGF-A189 is potent in inducing fibroblast proliferation [2, 18]. VEGF-189 and VEGF-206 are sequestered mainly in the extracellular matrix due to possessing heparin-binding domain [5].

Placental growth factor and VEGF-B are the next family members. They act by binding only to VEGFR-1 (Flt1) [1, 4]. VEGF-B has a minor role in angiogenesis regulation, but it plays a role in cardiac muscle function and the coronary artery system $[1,3]$. Animal models show that it may be tissue specific, and that it may play a different role in different target organs [19]. PlGF, like other members of the VEGF family, induces angiogenesis, and increases vascular permeability and inflammation [19]. It acts as a proangiogenic factor, also by enhancing VEGF-A activity [19].

VEGF-C and VEGF-D have a very weak affinity to VEGFR-2, but they act mainly via VEGFR-3 (Flt4) receptor [1]. Consequently they play a minor role in angiogenesis, but they are critical regulators for lymphangiogenesis because VEGFR-3 is expressed on lymphatic endothelial cells [4, 16, 17]. Hence, VEFG-C/D-VEGFR3 signals may be involved in lymph-node tumour metastasis $[1,4]$. VEGF-C and its receptor - VEGFR3 - are essential for Schlemm's canal development [20]. In an adult animal model they induced proliferation and growth of Schemm's canal endothelial cells, and they reduced intraocular pressure (IOP) [20, 21].

VEGF-E is not encoded by humans, but by a viral gene from the orf virus. This parapoxvirus infects goats, sheep, and sometimes humans and induces the angiogenesis at sites of skin infection [4]. It was found that VEGF-E tightly binds and activates very specifically VEGFR-2 [4].

\section{Anti-VEGF therapy}

As VEGF-VEGFRs proteins play a central role in angiogenesis and are involved in so many pathological conditions, the idea of blocking these potent factors opened new possibilities in medicine. Bevacizumab, an anti-VEGF agent, not only inhibits vessel growth and neovascularisation, but also induces regression of pathological microvessels, stabilises normal vessels, and prevents leakage and concomitant inflammatory response [22].

The possibilities of the attenuation of VEGF function on three non-overlapping levels are listed below [11].

First, synthesis of this factor may be modified by blocking VEGF gene expression [11]. There are attempts to use small-interfering RNA: silencing RNA (siRNA) sequences that are able to silence the original gene due to mutual homology, and thus to inhibit VEGF synthesis and secretion $[11,16]$. These particles are able to penetrate across cellular membranes, and induce inhibition of post-transcriptional RNA processing, so they can reduce both intracellular and extracellular effects of VEGF [16]. Currently, siRNA therapeutic agents: bevasiranib and SIRNA-027, are being tested for neovascular AMD and corneal neovascularisation $[10,16]$.

The second option is direct VEGF blocking. Nowadays, this line already has a grounded position in medicine. Drugs acting in this way are:

- Bevacizumab (Avastin, Genentech, San Francisco, CA, USA), a full-length humanised recombinant monoclonal IgG anti-VEGF-A antibody. It binds and inhibits all VEGF-A isoforms [11, 23, 24]. Its molecular weight is $148 \mathrm{kDa}$, so it is a large molecule with twice the halflife of ranibizumab $[12,13]$. It has been approved for the treatment of several solid tumours (colorectal, non-epithelial lung, breast, ovarian, and renal cancers) and glioblastomas [3, 24-26]. In ophthalmology it is used as an off-label procedure [11, 12, 27, 28]. Furthermore, it is probably still the most widely used anti-VEGF drug in ophthalmology due to much lower costs of therapy, compared with other medicines [12, 24, 29].

- Ranibizumab (Lucentis, Genentech, San Francisco, CA, USA/Novartis Ophthalmics, Basel, Switzerland) is a (Fab) fragment of a humanised monoclonal anti VEGF-A antibody, also against all VEGF-A isoforms $[10,13,23]$. Its molecular weight is $48 \mathrm{kDa}$ [24]. This drug was designed for eye diseases, and it was approved for intra-ocular use in neovascular AMD, macular oedema (ME) after retinal vein occlusions (RVO), diabetic macular oedema (DME), and diabetic retinopathy (DR) with DME [30]. In any other ocular diseases it is also used off label. 
- Pegaptanib (Macugen, Phizer, New York), a 28-base ribonucleic acid aptamer, covalently linked to two branched 20-kd polyethylene glycol moieties [10, 23]. It specifically binds and blocks activity of extracellular VEGF-A165 isoform $[11,23]$. It was used in wet AMD treatment, but it was found to be weaker than the drugs listed above. This is probably due to its specificity for binding only one isoform of VEGF [16].

- Aflibercept (Eylea, Regeneron, Tarrytown, NY, USA), a VEGF-trap: a 115-kDa recombinant fusion decoy protein consisting of VEGF binding domains of human VEGFR-1 and VEGFR-2 fused to the Fc domain of human immunoglobulin G1 [23]. It binds all forms of VEGF-A but also PlGF-1 and PlGF-2 with a very high affinity, greater than bevacizumab or ranibizumab $[10,11,16]$. It was approved for colorectal metastasising carcinoma treatment (Zaltrap). In ophthalmology it has already been approved as a therapy for neovascular AMD, macular oedema after RVO, and diabetic macular oedema [31].

The third option is inhibition of VEGF signalling, for instance by receptor blocking. As all forms of the VEGF act by stimulation of specific tyrosine kinase receptors, inhibition of these kinases may result in suppression of their activity [11]. Multi-kinase inhibitors, such as sorafenib and sunitinib are approved in renal and hepatocellular cancer treatment, and there are attempts to use them in ophthalmology $[4,10]$. Another tyrosine kinase inhibitor, pazopanib (Votrient, GlaxoSmitheKline, Hertfordshire, UK) is tested for retinal disorders treatment [16]. It was also found that peroxisome proliferators-activated receptor $\gamma$ (PPAR- $\gamma$ ) agonists are able to inhibit VEGF signalling [11]. Rosiglitazone and pioglitazone, (PPAR- $\gamma$ agonists) were already used clinically to control diabetes, and rosiglitazone delayed the onset of proliferative retinopathy in type 2 diabetics [11].

\section{Anti-VEGF therapy in ophthalmology}

Increased expression of VEGF was found to be an important factor in pathogenesis of many ocular diseases [5]. There is no doubt that nowadays anti-VEGF therapy is a potent and effective weapon in the fight against neovascular age-related macular degeneration (AMD), complications of diabetic retinopathy, and retinal vein occlusions. Numerous independent studies have proven its usefulness and efficacy: the CRUISE and BRAVO studies for macular oedema after central retinal vein occlusion, the RESOLVE, RESTORE, READ-2, and DA VINCI studies for diabetic macular oedema, and the MARINA, ANCHOR, PIER, PRONTO, VISION, LEVEL, CATT, CLEAR-IT studies for age-related macular degeneration [10, 23, 32-34]. There are also attempts to apply these agents in the management of other forms of diabetic retinopathy, retinopathy of prematurity, and of choroidal neovascu- larisation caused by another eye diseases such as angioid streaks, pathologic myopia, traumatic choroidal rupture, or ocular histoplasmosis [11]. This therapy is used for corneal neovascularisation treatment, too $[16,35]$. Furthermore, it may be used as a potent adjuvant in glaucoma filtering surgery and in neovascular glaucoma management [5, $32,36]$. Anti-VEGF agents seem to be superior to certain modalities addressed to the diseases mentioned above. In light of current knowledge, they neither cause structural destructive changes in the retina, as lasers do, nor induce cataract formation as steroids do [11].

\section{Safety of anti-VEGF therapy}

The application of anti-VEGF therapy in ocular diseases management is still increasing. Fortunately, the adverse effects, both local and systemic, of this regiments are rare compared to the number of administrations [34, 37].

\section{Local adverse effects}

Many preclinical studies assessed the adverse effects of anti-VEGF therapy, finding them to be safe and non toxic when standard doses were administered [38]. In vivo studies reported the following:

IOP elevation. As shown above, anti-VEGF therapy is nowadays widely used in ophthalmology and in glaucoma management. However, paradoxically these drugs may be involved in glaucoma development or progression. Many studies reported cases of both short-term as well as sustained elevation of intraocular pressure after intraocular use of anti-VEGF agents [27].

- Immediate IOP elevation after intravitreal injection is usually temporary, and it is caused by a vitreous volume increase [34]. Numerous independent trials showed this phenomenon [27, 39-42]. Other studies demonstrated, that these peaks of IOP normally resolve quickly (from five minutes to one hour) [40, 42, 43]. They are compensated through an aqueous outflow increase [34]. Because in glaucoma eyes outflow facility is decreased, the normalisation of a post-injection IOP takes longer [34, 40, 42]. Some investigators assessed the relationship between the post-injection elevation of the IOP and the axial length [27]. They found a positive correlation between shorter axial length and higher post-injection IOP, but only during the 1 to 15 minutes after injection $[27,39,44]$. It was estimated that the rise in IOP after intravitreal bevacizumab (IVB) injection occurs in $0.16 \%$ of patients [13]. These peaks of IOP resolve within one hour, so IOP monitoring after intraocular injection is not a standard procedure $[27,43]$. On the other hand, severe elevations of the IOP in eyes with advanced glaucoma may be dangerous [34, 42]. For this reason some clinicians suggest not only to monitor this parameter in glaucoma patients but also to apply prophylaxis: IOP lowering drugs, Honan Ballon, or 
anterior chamber paracentesis after an intravitreal injection [27, 34, 42].

- Sustained IOP elevation. Initially clinical trials assessed anti-VEGF therapy as safe in respect to elevations of IOP in long-term observations [27, 42]. Unfortunately, with the passing of time an increasing number of reports about sustained elevation of IOP after this kind of treatment were seen in medical literature $[34,45]$. This is especially true for patients with preexisting glaucoma [46]. Sustained IOP elevation is defined very diversely, usually as IOP $>21-25 \mathrm{mmHg}$ or as an increase from baseline $\geq 5 \mathrm{mmHg}$ on two separate visits, requiring glaucoma medication or surgery [27, 34, 43]. This hampers the drawing of comparable conclusions [47]. In patients receiving anti-VEGF intravitreal injections for AMD treatment, the frequency of sustained IOP elevation was estimated at $3.45-11.6 \%$ [47]. Such an elevation is supposed to be induced in several different mechanisms. One of such proposed mechanism is a blockage of trabecular meshwork by drug particles. It may be true for large particles such as bevacizumab (148 kDa) particles [47]. This structure may also be blocked by silicone oil droplets from needles and syringes, used by manufacturers for lubrication of these devices [27, 48]. Moreover, bevacizumab before its distribution for clinical use is frequently repackaged by compounding pharmacies, and this causes the formation of of high-molecular-weight aggregates of dimers and trimers of bevacizumab IgG monomers [27, $42,48,49]$. Trabeculitis or subclinical uveitis, induced by anti-VEGF drugs, are the other proposed causes [34, 42, $47,50,51]$. A potential direct toxic effect of anti-VEGF drugs on trabecular meshwork cells was also proposed [52]. However, Kahook et al. in a in vitro model of human trabecular meshwork cells observed the reduction of cells metabolism and proliferation, only after exposure for high concentrations of bevacizumab [52]. No changes for ranibizumab, and lower concentrations of bevacizum$\mathrm{ab}$ and components of these drugs vehicles were observed [52]. As these cells express Fc receptors, the intracellular accumulation of antibodies may be present. A higher number of intravitreal injections was found to be an independent factor for sustained elevation of IOP, although it was not proven in other studies [27, 47, 53-56]. Patients with preexisting glaucoma or ocular hypertension also seem to be more susceptible [34, 47, 57, 58]. Reports assessing the correlation between the sustained IOP after anti-VEGF therapy and the patient's demographics or drug type are contradictory [34]. Although sustained IOP after anti-VEGF therapy is usually controlled by locally applied eye drops, some authors reported a need for laser or even surgical treatment in this group [27, 34, 59].

Other ocular complications such as a vitreous haemorrhage, retinal tear formation, tractional retinal detachment, macular hole formation, or endophthalmitis seem to be rather injection related because the fluctuations in IOP and deformation of the globe during intravitreal injection may result in vitreoretinal traction $[5,13]$. The presence of severe proliferative vitreoretinopathy is a risk factor for tractional retinal detachment in these eyes [13]. It is also supposed that anti-VEGF therapy may switch angiogenesis to fibrosis an thus increase retinal traction [13]. In eyes without such predisposing factors (treated for AMD only) the occurrence of RD was reported in $0.01-0.08 \%$ per injection [60]. The occurrence of bacterial endophthalmitis after anti-VEGF intravitreal injections varies from study to study in the range $0.05-1.2 \%$ per injection $[13,22,60]$. Also, infrequently cases of noninfectious uveitis, especially after IVB, were reported $[5,61]$. The incidence of this adverse effect ranged from $1.4 \%$ to $2.9 \%$ after intravitreal ranibizumab (IVR) and from $0.09 \%$ to $1.3 \%$ after IVB injections [5]. Data showing higher incidence of cataract formation and progression are conflicting, but its rate is probably low [60]. Some studies rise also the issue of potential risk for the increase of the macular ischaemia by foveal avascular zone (FAZ) enlargement after anti-VEGF therapy $[5,13]$.

The Collaborative Anti-VEGF Ocular Vascular Complications Group assessed vascular events following injections of anti-VEGF agents [37]. They analysed the frequency of vascular occlusions such as: central retinal artery occlusion (CRAO), branch retinal artery occlusion (BRAO), capillary occlusions, branch retinal vein occlusion (BRVO), central retinal vein occlusion (CRVO), anterior ischaemic optic neuropathy (AION), and ocular ischaemic syndrome after anti-VEGF therapy [37]. The reports from these studies revealed that the overall risk of ocular vascular events after injection of VEGF antagonist was estimated at $0.108 \%$ in the general study population, and at $2.61 \%$ in the diabetic population [37]. Higashide $e t$ al. analysed the occurrence of adverse events after IVB in patients with neovascular glaucoma. They reported CRAO in $2 \%$, mainly in eyes with preexisting ocular ischaemic syndrome, but they did not find cases with other intraocular or systemic adverse effects [62]. It suggests that vascular events reported during anti-VEGF therapy may be a part of the natural history of the treated ocular disease [5]. Preexisting risk factors for ischaemic eye diseases and cardiovascular events were present in the majority of patients included in these studies [37, 62].

After injection of anti-VEGF agents directly to the anterior chamber or after their penetration following intravitreal injection to the anterior segment of the eye, the risk for endothelial cell damage and corneal oedema is obvious. In animal models neither bevacizumab nor ranibizumab given intracamerally had a damaging effect on the corneal endothelium [63]. Rusovici et al. assessed the influence of bevacizumab on VEGF-enriched corneal endothelial cells. They found that this drug in conventional doses did not show the cytotoxicity and did not change cell proliferation and morphology [64]. 


\section{Systemic adverse side effects}

The risk of systemic side effects of anti-VEGF therapy was known before its usage in ophthalmology. Bavacizumab was already applied intravenously for malignant tumour treatment, and the possibility of hypertension, proteinuria, thromboembolic events with strokes, myocardial infarctions, gastroinstetinal perforations, and even death was well documented [5, 10, 37]. However, serum levels of anti-VEGF drugs after intravitreal injections are incomparably lower than after intravenous administration [5, 10]. In a Pan-American Collaborative Retina Study Group (PACORES) report on patients receiving IVB injections an acute blood pressure elevation was observed in $0.59 \%$, myocardial infarction in $0.4 \%$, cerebrovascular accidents in $0.5 \%$, and death in $0.42 \%$ [5, 65]. In a meta-analysis by Ueta et al. the incidence of all cardiovascular accidents: transient ischaemic attacks, strokes, and other cerebral ischaemic incidents was $2.2 \%$ in patients treated with IVR injections, versus $0.7 \%$ in the sham-treated ones [5, 66]. There was no association between IVR injections and myocardial infarcts $[5,66]$.

\section{Conclusions}

Nowadays, anti-VEGF therapy has become one of the more frequently applied treatment modalities in ophthalmology. The indications for its usage have become wider and wider, but investigators still are looking for new drugs with more prolonged time of action or new forms of drugs delivery.

The authors declare no conflict of interest.

\section{References}

1. Shibuya M (2014): VEGF-VEGFR signals in health and disease. Biomol Ther (Seoul) 22: 1-9.

2. Van Bergen T, Vandewalle E, Van de Veire S, et al. (2011): The role of different VEGF isoforms in scar formation after glaucoma filtration surgery. Exp Eye Res 93: 689-699.

3. Shibuya M (2013): Vascular endothelial growth factor and its receptor system: physiological functions in angiogenesis and pathological roles in various diseases. J Biochem 153: 13-19.

4. Shibuya M (2011): Vascular endothelial growth factor (VEGF) and its receptor (VEGFR) signaling in angiogenesis: a crucial target for anti- and pro-angiogenic therapies. Genes Cancer 2: 1097-1105.

5. Tolentino M (2011): Systemic and ocular safety of intravitreal anti-VEGF therapies for ocular neovascular disease. Surv Ophthalmol 56: 95-113.

6. Chalam KV, Brar VS, Murthy RK (2014): Human ciliary epithelium as a source of synthesis and secretion of vascular endothelial growth factor in neovascular glaucoma. JAMA Ophthalmol 132: 1350-1354.

7. Kaur C, Foulds WS, Ling EA (2008): Hypoxia-ischemia and retinal ganglion cell damage. Clin Ophthalmol 2: 879-889.
8. Kaur C, Sivakumar V, Foulds WS (2006): Early response of neurons and glial cells to hypoxia in the retina. Invest Ophthalmol Vis Sci 47: 1126-1141.

9. Kim M, Lee C, Payne R, et al. (2015): Angiogenesis in glaucoma filtration surgery and neovascular glaucoma: A review. Surv Ophthalmol 60: 524-535.

10. Andreoli CM, Miller JW (2007): Anti-vascular endothelial growth factor therapy for ocular neovascular disease. Curr Opin Ophthalmol 18: 502-508.

11. Al-Latayfeh M, Silva PS, Sun JK, Aiello LP (2012): Antiangiogenic therapy for ischemic retinopathies. Cold Spring Harb Perspect Med 2: a006411.

12. Popescu V, Pricopie S, Totir M, et al. (2015): Clinical use of Bevacizumab in treating refractory glaucoma. J Med Life 8: 8-12.

13. Osaadon P, Fagan XJ, Lifshitz T, Levy J (2014): A review of anti-VEGF agents for proliferative diabetic retinopathy. Eye (Lond) 28: 510-520.

14. Foxton RH, Finkelstein A, Vijay S, et al. (2013): VEGF-A is necessary and sufficient for retinal neuroprotection in models of experimental glaucoma. Am J Pathol 182: 1379-1390.

15. Noma H, Mimura T, Yasuda K, Shimura M (2014): Vascular endothelial growth factor and its soluble receptors- 1 and -2 in iris neovascularization and neovascular glaucoma. Ophthalmologica 232: 102-109.

16. Chang JH, Garg NK, Lunde E, et al. (2012): Corneal neovascularization: an anti-VEGF therapy review. Surv Ophthalmol 57: 415-429.

17. Zheng W, Aspelund A, Alitalo K (2014): Lymphangiogenic factors, mechanisms, and applications. J Clin Invest 124: 878887.

18. Daneshvar R (2013): Anti-VEGF agents and glaucoma filtering surgery. J Ophthalmic Vis Res 8: 182-186.

19. Chen S, Zhou M, Wang W, et al. (2015): Levels of angiogenesis-related vascular endothelial growth factor family in neovascular glaucoma eyes. Acta Ophthalmol 93: e556-560.

20. Aspelund A, Tammela T, Antila S, et al. (2014): The Schlemm's canal is a VEGF-C/VEGFR-3-responsive lymphatic-like vessel. J Clin Invest 124: 3975-3986.

21. Karpinich NO, Caron KM (2014): Schlemm's canal: more than meets the eye, lymphatics in disguise. J Clin Invest 124: 37013703.

22. Klein A, Loewenstein A (2016): Therapeutic monoclonal antibodies and fragments: Bevacizumab. Dev Ophthalmol 55: 232-245.

23. Kimoto K, Kubota T (2012): Anti-VEGF agents for ocular angiogenesis and vascular permeability. J Ophthalmol 2012: 852183.

24. Park SC, Su D, Tello C (2012): Anti-VEGF therapy for the treatment of glaucoma: a focus on ranibizumab and bevacizumab. Expert Opin Biol Ther 12: 1641-1647.

25. Horsley MB, Kahook MY (2010): Anti-VEGF therapy for glaucoma. Curr Opin Ophthalmol 21: 112-117.

26. http://www.ema.europa.eu/docs/pl_PL/document_library/ EPAR_-_Product_Information/human/000582/WC500029271. pdf-14.12.2015

27. SooHoo JR, Seibold LK, Kahook MY (2014): The link between intravitreal antivascular endothelial growth factor injections and glaucoma. Curr Opin Ophthalmol 25: 127-133.

28. Ryu CL, Elfersy A, Desai U, et al. (2014): The effect of antivascular endothelial growth factor therapy on the development of neovascular glaucoma after central retinal vein occlusion: a retrospective analysis. J Ophthalmol 2014: 317694. 
29. Cardarelli WJ, Smith RA (2013): Managed care implications of age-related ocular conditions. Am J Manag Care 19 (5 Suppl): S85-91.

30. http://www.ema.europa.eu/docs/pl_PL/document_library/EPAR_-_Summary_for_the_public/human/002392/ WC500135745.pdf-14.12.2015

31. http://www.ema.europa.eu/docs/pl_PL/document_library/ EPAR_-_Product_Information/human/002532/WC500139484. pdf -14.12 .2015$

32. Simha A, Braganza A, Abraham L, et al. (2013) Anti-vascular endothelial growth factor for neovascular glaucoma. Review Cochrane Database Syst Rev 10: CD007920.

33. Akpek EK, Smith RA (2013): Current treatment strategies for age-related ocular conditions. Am J Manag Care 19 (5 Suppl): S76-84.

34. Singh RS, Kim JE (2012): Ocular hypertension following intravitreal anti-vascular endothelial growth factor agents. Drugs Aging 29: 949-956.

35. Bahar I, Yeung SN, Sella R, Slomovic A (2012): Anterior segment uses of bevacizumab. Curr Opin Ophthalmol 23: 303-316.

36. Xiong Q, Li Z, Li Z, et al. (2014): Anti-VEGF agents with or without antimetabolites in trabeculectomy for glaucoma: a meta-analysis. PLoS One 9: e88403.

37. Mansour AM, Shahin M, Kofoed PK, et al. (2012): Collaborative Anti-VEGF Ocular Vascular Complications Group. Insight into 144 patients with ocular vascular events during VEGF antagonist injections. Clin Ophthalmol 6: 343-363.

38. SooHoo JR, Seibold LK, Kahook MY (2013): Recent advances in the management of neovascular glaucoma. Semin Ophthalmol 28: 165-172.

39. Gismondi M, Salati C, Salvetat ML, et al. (2009): Short-term effect of intravitreal injection of Ranibizumab (Lucentis) on intraocular pressure. J Glaucoma 18: 658-661.

40. Kim JE, Mantravadi AV, Hur EY, Covert DJ (2008): Shortterm intraocular pressure changes immediately after intravitreal injections of anti-vascular endothelial growth factor agents. Am J Ophthalmol 146: 930-4.e1.

41. Hollands H, Wong J, Bruen R, et al. (2007): Short-term intraocular pressure changes after intravitreal injection of bevacizumab. Can J Ophthalmol 42: 807-811.

42. Aref AA (2012): Management of immediate and sustained intraocular pressure rise associated with intravitreal antivascular endothelial growth factor injection therapy. Curr Opin Ophthalmol 23: 105-110.

43. Kiddee W, Montriwet M (2015): Intraocular pressure changes in non-glaucomatous patients receiving intravitreal anti-vascular endothelial growth factor agents. PLoS One 10: e0137833.

44. Cacciamani A, Oddone F, Parravano M, et al. (2013): Intravitreal injection of bevacizumab: changes in intraocular pressure related to ocular axial length. Jpn J Ophthalmol 57: 63-67.

45. Choi DY, Ortube MC, McCannel CA, et al. (2011): Sustained elevated intraocular pressures after intravitreal injection of bevacizumab, ranibizumab, and pegaptanib. Retina 31: 1028-1035.

46. Good TJ, Kimura AE, Mandava N, Kahook MY (2011): Sustained elevation of intraocular pressure after intravitreal injections of anti-VEGF agents. Br J Ophthalmol 95: 1111-1114.

47. Dedania VS, Bakri SJ (2015): Sustained elevation of intraocular pressure after intravitreal anti-VEGF agents: what is the evidence? Retina 35: 841-858.

48. Bakri SJ, Ekdawi NS (2008) Intravitreal silicone oil droplets after intravitreal drug injections. Retina 28: 996-1001.

49. Kahook MY, Liu L, Ruzycki P, et al. (2010): High-molecular-weight aggregates in repackaged bevacizumab. Retina 30 : 887-892.
50. Sniegowski M, Mandava N, Kahook MY (2010): Sustained intraocular pressure elevation after intravitreal injection of bevacizumab and ranibizumab associated with trabeculitis. Open Ophthalmol J 4: 28-29.

51. Bakri SJ, McCannel CA, Edwards AO, Moshfeghi DM (2008): Persistent ocular hypertension following intravitreal ranibizumab. Graefes Arch Clin Exp Ophthalmol 246: 955-958.

52. Kahook MY, Ammar DA (2010): In vitro effects of antivascular endothelial growth factors on cultured human trabecular meshwork cells. J Glaucoma 19: 437-441.

53. Bakri SJ, Moshfeghi DM, Francom S, et al. (2014): Intraocular pressure in eyes receiving monthly ranibizumab in 2 pivotal age-related macular degeneration clinical trials. Ophthalmology 121: 1102-1108.

54. Tseng JJ, Vance SK, Della Torre KE, et al. (2012): Sustained increased intraocular pressure related to intravitreal antivascular endothelial growth factor therapy for neovascular age-related macular degeneration. J Glaucoma 21: 241-247.

55. Hoang QV, Mendonca LS, Della Torre KE, et al. (2012): Effect on intraocular pressure in patients receiving unilateral intravitreal anti-vascular endothelial growth factor injections. Ophthalmology 119: 321-326.

56. Kim YJ, Sung KR, Lee KS, et al. (2014): Long-term effects of multiple intravitreal antivascular endothelial growth factor injections on intraocular pressure. Am J Ophthalmol 157: 12661271.

57. Kahook MY, Kimura AE, Wong LJ, et al. (2009): Sustained elevation in intraocular pressure associated with intravitreal bevacizumab injections. Ophthalmic Surg Lasers Imaging 40: 293-295.

58. Kim YJ, Sung KR, Lee KS, et al. (2014): Long-term effects of multiple intravitreal antivascular endothelial growth factor injections on intraocular pressure. Am J Ophthalmol 157: 12661271.

59. Skalicky SE, Ho I, Agar A, Bank A (2012): Glaucoma filtration surgery following sustained elevation of intraocular pressure secondary to intravitreal anti-VEGF injections. Ophthalmic Surg Lasers Imaging 43: 328-334.

60. Sampat KM, Garg SJ (2010): Complications of intravitreal injections. Curr Opin Ophthalmol 21: 178-183.

61. Bakri SJ, Larson TA, Edwards AO (2008): Intraocular inflammation following intravitreal injection of bevacizumab. Graefes Arch Clin Exp Ophthalmol 246: 779-781.

62. Higashide T, Murotani E, Saito Y, et al. (2012): Adverse events associated with intraocular injections of bevacizumab in eyes with neovascular glaucoma. Graefes Arch Clin Exp Ophthalmol 250: 603-610.

63. Wilhelm A, Sietmann R, Wilhelm U, Hammer T (2015): The influence of VEGF inhibitors on corneal endothelium after injection into the anterior chamber in a porcine eye model. Ophthalmologe 112: 346-352.

64. Rusovici R, Sakhalkar M, Chalam KV (2011): Evaluation of cytotoxicity of bevacizumab on VEGF-enriched corneal endothelial cells. Mol Vis 17: 3339-3346.

65. Wu L, Martínez-Castellanos MA, Quiroz-Mercado H, et al. (2008): Pan American Collaborative Retina Group (PACORES). Twelve-month safety of intravitreal injections of bevacizumab (Avastin): results of the Pan-American Collaborative Retina Study Group (PACORES). Graefes Arch Clin Exp Ophthalmol 246: 81-87.

66. Ueta T, Yanagi Y, Tamaki Y, Yamaguchi T (2009): Cerebrovascular accidents in ranibizumab. Ophthalmology 116: 362. 